\title{
Simulations of Multiphase Flow in Naturally Heterogeneous Rocks
}

\author{
PATERSON, Lincoln, CSIRO Petroleum \\ PAINTER, Scott, CSIRO Petroleum \\ ZHANG, Xiaodong, University of New South Wales \\ PINCZEWSKI, W. Val, University of New South Wales
}

Paper presented at the 5th European Conference on the Mathematics of Oil Recovery, Leoben, Austria, 3-6 Sept. 1996

\begin{abstract}
In this paper we present results of network flow simulations with heterogeneity corresponding to naturally occurring sedimentary rocks. Correlations were generated using fractional Brownian motion, fractional Lévy motion and multifractals. Flow simulations were based on two-phase capillary driven invasion percolation in three dimensional networks. Results for breakthrough saturation, residual saturation and relative permeability curves for correlated networks are compared to uncorrelated networks
\end{abstract}

With correlated heterogeneity, relative permeability is greater when flow is parallel to the bedding rather than perpendicular. Variability for a given statistical description is also greater in the correlated media.

\section{INTRODUCTION}

Understanding multiphase flow in naturally occurring heterogeneous sedimentary rock is vital to performance prediction in the petroleum industry. Results from reservoir simulation are sensitive to input parameters that include residual saturation and relative permeability. Values for residual saturation and relative permeability are normally obtained from empirical laboratory measurements or history matching of field behaviour. A direct theoretical understanding only appeared with the advent of network models and percolation theory. Network models have possibly been the most successful tool for understanding multiphase flow in porous media.

Network models have generally been implemented with spatially uncorrelated properties. These models include percolation and its derivatives, as well as the diffusion- limited aggregation model for viscous fingering and its derivatives. Increasingly sophisticated network models now exist [eg. Pereira et al. 1996].

Common methods of interpreting laboratory measurements require the assumption of homogeneity within the sample. However, for practical reasons, many cores that are tested are heterogeneous to varying degrees. Likewise, petroleum reservoirs exhibit varying degrees of large-scale heterogeneity.

One of the challenges to incorporating heterogeneity into network models has been deriving a theoretical description that is both versatile and accurate. Significant advances in our ability to quantitatively describe property variations in natural rocks have been recently reported. A description based on fractional Lévy motion has been found to be consistent with data from many wells and outcrop measurements [Painter and Paterson 1994; Painter 1995, 1996a]. Fractional Brownian motion, which has also been used for sedimentary rock [Hewitt 1985], is a special case of fractional Lévy motion, and both are examples of a statistical fractal description. This paper describes the incorporation of an effective heterogeneity model into network simulations. The results lead to an understanding of a number of important previously reported observations concerning the complex effect of heterogeneity on residual saturations and relative permeability in laboratory tests of reservoir cores.

\section{NETWORK MODELS}

Network models have been described as the best tool for understanding multiphase flow in porous media. Usually network models subdivide the pore space into two 
components: pores and throats [eg. Jerauld and Salter 1990; Blunt and King 1991; Pereira et al. 1996]. Pores contain most of the open volume available to fluid and the throats are the constrictions that subdivide the pores. However, these definitions are not rigorous. For instance, throats can be associated with resistance to flow and interpreted in terms of the contribution to permeability via connected tubes of equivalent radii. Alternatively they can be defined as the minimum radius that exists for a meniscus to pass through as it moves from pore to pore. Subdivision into pores and throats is not essential for a working network model.

The most basic network models are based on percolation theory [Larson et al. 1977, 1981; Wilkinson and Willemsen 1983; Heiba et al. 1992; Sahimi 1993]. Blunt et al. [1992] have discussed the relevance of invasion percolation and percolation models to various capillary dominated modes of flow that include drainage and imbibition. The present results are primarily concerned with invasion percolation, corresponding to the displacement of a wetting fluid by a non-wetting fluid. At each step in the invasion process, the next largest site adjacent to the invading fluid is occupied. Obtaining relative permeability curves from percolation models is best performed on a three-dimensional network, because true two-phase flow does not occur in twodimensions [Blunt et al. 1992].

In this paper we only use a single component site model, which can be interpreted as a set of pairs of pores and throats that occupy each site. Using this abstraction simplifies the numerical implementation. This has benefits in studies of large three-dimensional networks where computation efficiency is an issue, and we have used 1000 realisations for most of the results presented in this paper. Furthermore, despite the importance of coordination number [Pathak et al. 1982], we confine ourselves to a simple cubic network of sites. These structural simplifications still allow an adequate qualitative test of the effects of heterogeneity, although refinement of the network model may be required for accurate quantitative predictions. Comparison of existing results for simple percolation models to more complex pore and throat models suggest that these differences may be small (Table 1).

Table 1. Percolation thresholds and residual saturation on 2 dimensional square networks. (a) results summarised in Hughes [1996] (b) from Pereira et al. [1996], 50 x 50 lattice.

$\begin{array}{ll}\text { bond percolation } & 0.50^{\text {(a) }} \\ \text { site percolation } & 0.59^{\text {(a) }} \\ \text { pore-throat model } & 0.60^{\text {(b) }}\end{array}$

The site invasion percolation model concerns site occupancy and site ranks. Site occupancy can be related to saturation of each of the fluid phases. Site rank can be related to pore throat radius. Macroscopic characterisation of porous media is normally done in terms of porosity and permeability, so it is necessary to interpret site occupancy in terms of porosity available to each fluid. Likewise, pore throat radii are connected to permeability.

In our simple model every pore is assigned identical volume, thus fraction of sites occupied in a percolation model becomes the fluid saturation. This assumption will be valid for media with a narrow range of porosity. However, pore sizes may vary significantly and be correlated. Similarly, every throat has been assigned equal conductivity, independent of radius. Some simulations were performed to test the consequences of this assumption.

The simulations presented here were conducted on cubic lattices of $L \times L \times L$ sites. Flow was from one face to the opposite, with no-flow boundary conditions applied on the remaining faces. Other published invasion percolation results have been obtained on $L \times L \times 2 L$ networks with periodic boundary conditions. Our studies in 2 dimensions have shown that these differences introduce small changes in the numerical values of the results, but there is no substantive change in behaviour.

It is convenient to work with $L \times L \times L$ networks with noflow boundaries when generating heterogeneous substrates. Periodic boundary conditions would also require the heterogeneity to be periodic. Relative permeability was calculated by solving Laplace's equation in each of the phases separately. Successive over-relaxation was used as the solution method.

The network model was implemented so that trapping could be turned on or off. Trapping occurs when a site containing defending fluid becomes disconnected from the outflow face. The three-dimensional trapping algorithm is very expensive in computer time and considerably limits the size of network. when turned on.

\section{HETEROGENEITY}

Increasingly accurate statistical descriptions of heterogeneity in sedimentary rocks have become available through the analysis of borehole data and studies of outcrops. Fractional Brownian motion $(\mathrm{fBm})$ was introduced as a description of reservoir heterogeneity by Hewitt [1985]. More recently a description based on the more general fractional Lévy motion (fLm) has been found to be more accurate [Painter and Paterson 1994; Painter 1995]. The fLm 


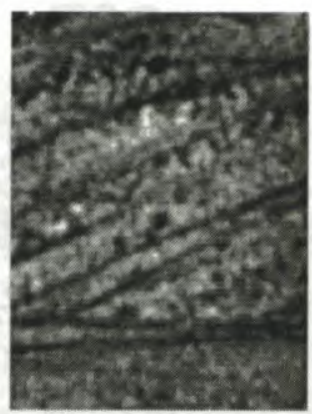

Figure 1. X-ray CT slice through a cross-bedded sandstone core. Correlated heterogeneity and anisotropy down to the limit of resolution $(\sim 1 \mathrm{~mm})$ can be seen.

model is parameterised by the Lévy index $\alpha$ and the Hurst parameter $H$. The Lévy index $0<\alpha \leq 2$ quantifies the degree of spatial variability, with increasing $\alpha$ implying increasing variability. The situation $\alpha=2$ is the special case of $\mathrm{fBm}$. The Hurst parameter $0<H<1$ quantifies the degree of spatial correlation; increasing $H$ implies increasing correlation. A feature of these fractional motion statistical descriptions is that they contain long range correlations in property values. This contrasts with the usual network models and glass micromodel simulations in which adjacent properties are independent.

The fLm model has been tested from the kilometre scale to scales as small as $0.03 \mathrm{~m}$. X-ray CT scanning of laboratory core samples often reveals laminations and other heterogeneities at scales down to about $1 \mathrm{~mm}$, which is the limit of resolution for most common scanners. An example of this small-scale heterogeneity is shown in Figure 1. Although the fLm model has not been tested down to this scale or the pore scale, it provides a convenient model for studying the effects of small-scale correlations and heterogeneities on two-phase displacements.

The heterogeneity maps corresponding to the fractional Lévy motion model were generated using a method devised by Painter [1996c]. This method is based on a multivariate generalisation of the Lévy distribution to model the multipoint joint distribution. This multiLevy method is more accurate in reproducing the statistical features observed in analyses of core and outcrop data compared with more approximate methods [Painter 1996b] for Lévy simulation.

For the special case of fractional Brownian motion $(\alpha=2$ in the Lévy model), another method based on the one described by Saupe [1988] was used. We also include some results for a multifractal property map for comparison. To generate the multifractal map, we extended to 3 dimensions the method described by Meakin [1987]. Anisotropic maps were

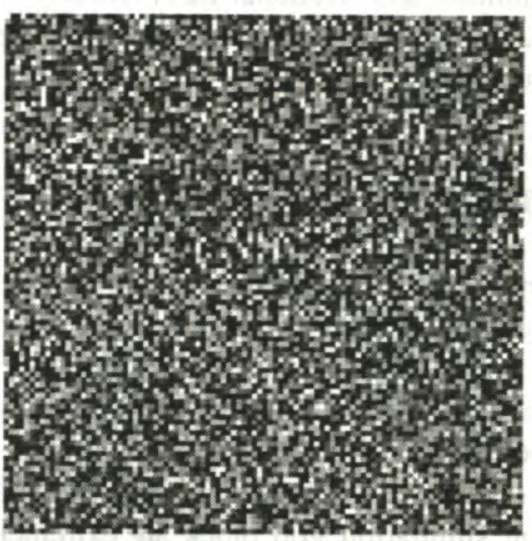

(a)

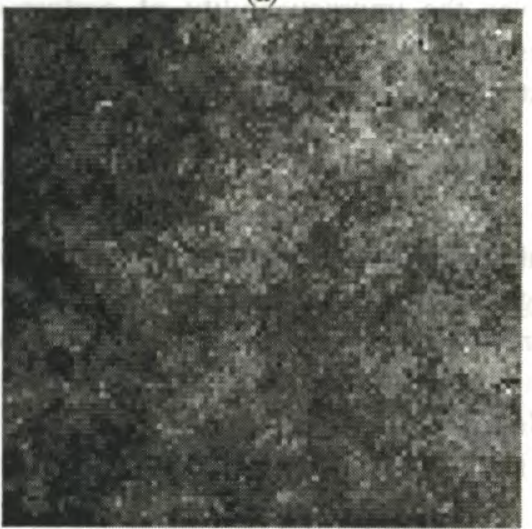

(b)

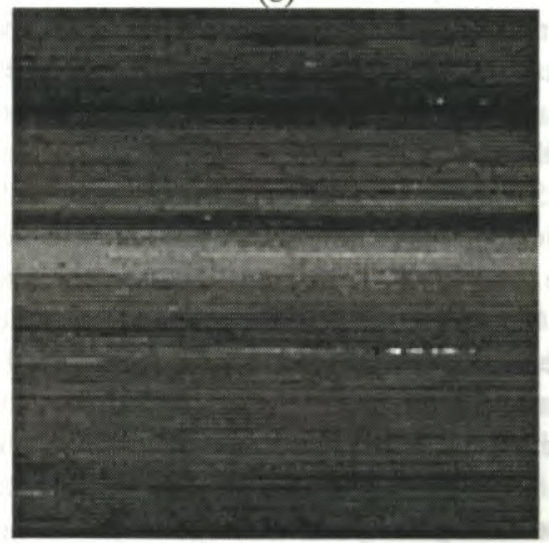

(c)

Figure 2. Property map generated (a) without correlations, (b) using fractional Brownian motion with $H=0.25$, and (c) using fractional Lévy motion with $\alpha=1.5, H=0.25$ and a horizontal stretching factor of 100 .

generated by compression in the vertical direction (equivalent to stretching in the horizontal direction). Examples of the appearance of various property maps are shown in Figure 2. Note that the finite size of the simulations means that the methods we have used only generate approximate fractional 
Brownian motion and fractional Lévy motion. Some of the precise detail in the numerical results may depend on the choice of method.

\section{VARIABILITY AND UPSCALING}

A laboratory core sample may contain about $10^{14}$ pores, whereas a petroleum reservoir may have in the order of $10^{30}$ pores. The networks simulations discussed in this paper range from about $10^{2}$ to $10^{5}$ sites, many orders of magnitude below core sizes. Scaling from the network models to laboratory core sizes, and from laboratory cores to the size of the reservoir simulation grid block are extremely important issues. Given the impracticability of performing network simulations with $10^{30}$ pores, well behaved scaling behaviour is an essential feature of a successful network model.

The term "upscaling" is normally interpreted to mean translating information on short length scales to the longer length scales of the reservoir simulation grid block. For instance, geostatistical methods are capable of providing many more values than can be easily accommodated in reservoir simulators, hence the geostatistically derived values are upscaled. Various methods of upscaling have been proposed [King 1989; Durlofsky 1991; Pickup and Sorbie 1994].

Another important practical upscaling problem occurs when a small set of laboratory measurements need to be interpreted at the scale of the reservoir. To illustrate, some actual residual oil saturation values from core testing in a well are shown in Table 2 . These values range from $22 \%$ to $42 \%$. The question is: what residual saturation value is correct to use for the reservoir? Furthermore, laboratory measurements may be biased toward homogeneous samples because heterogeneities are avoided during core-plug screening [Honarpour et al. 1995]. One reason for this is that methods of interpreting transient relative permeability tests such as the JBN method [Johnson et al. 1959] involve the assumption that the core is homogeneous. It then becomes necessary to translate the laboratory results from the homogeneous cores to a possibly heterogeneous reservoir. The selection of homogeneous cores may lead to a biased interpretation at the reservoir level.

Table 2. Example of residual oil saturation values from core testing. These cores were obtained from a single well.
0.22
0.29
0.40
0.22
0.32
0.42

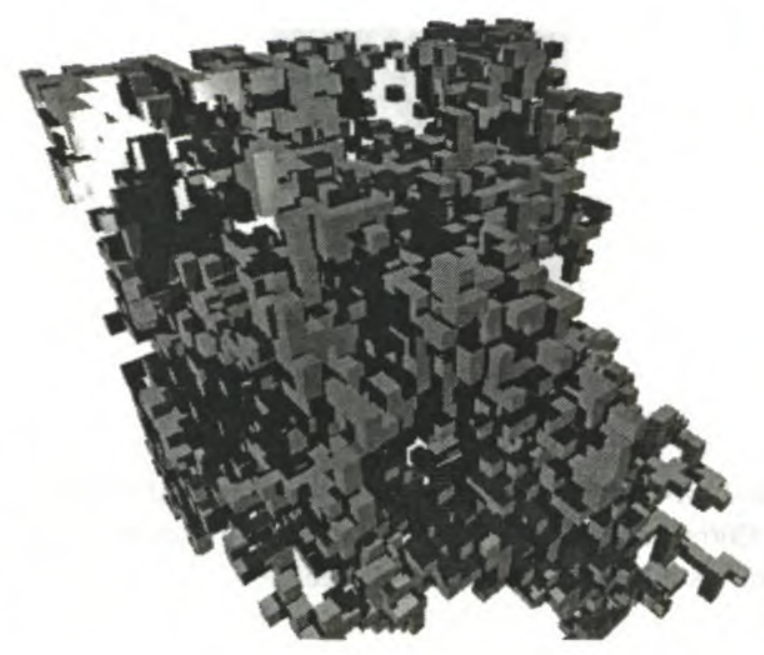

Figure 3. Examples of the invading fluid at initial breakthrough for site invasion percolation on an uncorrelated network.

Honarpour et al. (1995) provide some discussion on scaleup. They consider the effect of small-scale laminations on relative permeability, and propose a method of characterisation based on X-ray CT scanning. The fractal $\mathrm{fBm}$ and fLm models imply that heterogeneity appears on all length scales.

\section{RESULTS}

Simulations were performed on a variety of network sizes up to $67 \times 67 \times 67$. The appearance of a typical invasion cluster is shown in Figure 3.

From percolation theory, the saturation $S_{b}$ at initial breakthrough of the invading fluid scales as

$$
S_{b} \sim L^{d}
$$

where $L$ is system size. On $L \times L \times L$ networks with uncorrelated properties we find $d=-0.45$. This compares with Wilkinson and Willemsen's [1983] value of -0.48 on $L \times L \times 2 L$ networks. For $\mathrm{fBm}$ with $H=0.5$, we find $d=-0.41$, so the correlated networks are less sensitive to size.

In an experimental study of breakthrough as a function of sample size, Hirsch and Thomson [1995] found that breakthrough for Berea sandstone scaled as $d \sim-0.5$. However, in Coconino sandstone, they find a value of $d$ much closer to -0.1 . This supports the notion that 
heterogeneity decreases the sensitivity to breakthrough in sample size.

We now address residual saturation of the defending phase. This required turning on the trapping algorithm in the network simulations. The more correlated the heterogeneity, the smaller the residual saturation (Table 3 ). The multifractal gives a result between uncorrelated and fractional Brownian motion. In two dimensions it is much easier to trap fluid than in three dimensions. This can be seen in the difference in the results between Tables 1 and 3 .

Table 3. Residual saturation values from site invasion percolation with trapping in $3 \mathrm{D}$ using various correlated heterogeneities. Isotropic correlations. The fLm example has $\alpha=1.5$.

$\begin{array}{ll}\text { uncorrelated } & 0.35 \\ \text { multifractal } & 0.31 \\ \text { fBm } H=0.1 & 0.29 \\ \text { fBm H=0.25 } & 0.28 \\ \text { fBm H=0.5 } & 0.27 \\ \text { fLm H=0.5 } & 0.27\end{array}$

The effect of network size on the residual saturation value $S_{r}$ obtained is shown in Figure 4. For sizes above $L=10$, little change in $S_{r}$ is observed (this provides the values for Table 3 above). Also shown in Figure 4 is a measure of the variability between individual realisations shown as \pm one standard deviation determined over 1000 realisations. The variability is much larger in the correlated networks, and appears to decrease with size. Simulations on much larger networks may be required to verify large-scale behaviour.

Table 4. Residual saturation values from site invasion percolation with trapping in 3D using correlated heterogeneities. Anisotropic correlations, with flow parallel or perpendicular to the bedding. The fLm examples have $\alpha=1.5$.

$\mathrm{fBm} \mathrm{H}=0.5$ stretch $=2$ parallel

$\mathrm{fBm} \mathrm{H}=0.5$ stretch $=2$ perpendicular 0.37

$\mathrm{fBm} \mathrm{H}=0.25$ stretch $=4$ parallel $\quad 0.23$

$\mathrm{fLm} \mathrm{H}=0.25$ stretch $=10$ parallel $\quad 0.20$

fLm $\mathrm{H}=0.25$ stretch $=100$ parallel $\quad 0.17$

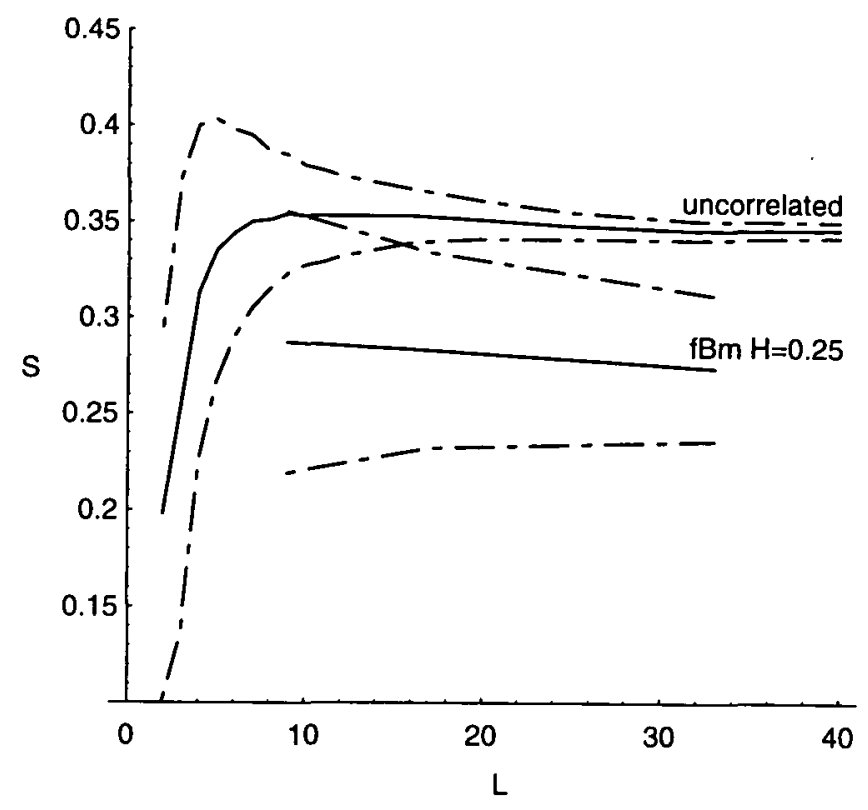

Figure 4. Effect of network size $L$ on residual saturation (a) an uncorrelated network, and (b) a fBm network with $H=0.25$. Also shown by broken lines are \pm one standard deviation determined from 1000 realisations at each value of $L$.

Residual saturation values in anisotropic networks are shown in Table 4. It is necessary to introduce correlations before bedding can be considered. Bedding corresponds to anisotropic correlations. Residual saturation for flow perpendicular to the bedding is substantially greater than for flow parallel to the bedding. The greater the anisotropy, the greater the effect.

Now we consider relative permeability while turning off trapping. Simulations showed that this makes little difference to the positions of the relative permeability curves. With trapping the curves are truncated at the residual saturation terminal point. Without trapping, simulations can be continued till the network is completely full of invading phase.

Some relative permeability results are shown in Figure 5. Relative permeabilities are plotted as a function of invading phase saturation $S$. At a given value of saturation relative permeability is greater in a correlated network, fractional Brownian motion with $H=0.5$, than an uncorrelated network.

The effect of heterogeneity on drainage gas-oil relative permeability curves has been experimentally studied by Corey and Rathjens [1956]. They investigated flow parallel 


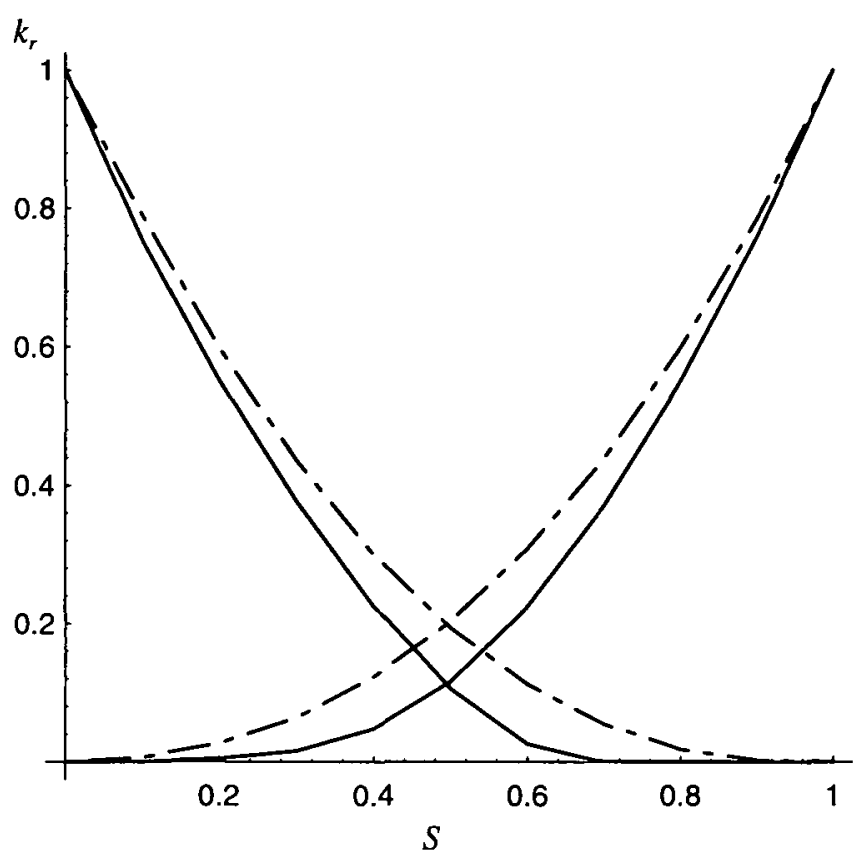

Figure 5. Relative permeability on an uncorrelated network (solid lines) compared against $\mathrm{fBm}$ with $H=0.5$ (broken lines). Simulations used $L=17$ with averages over 1000 realisations.

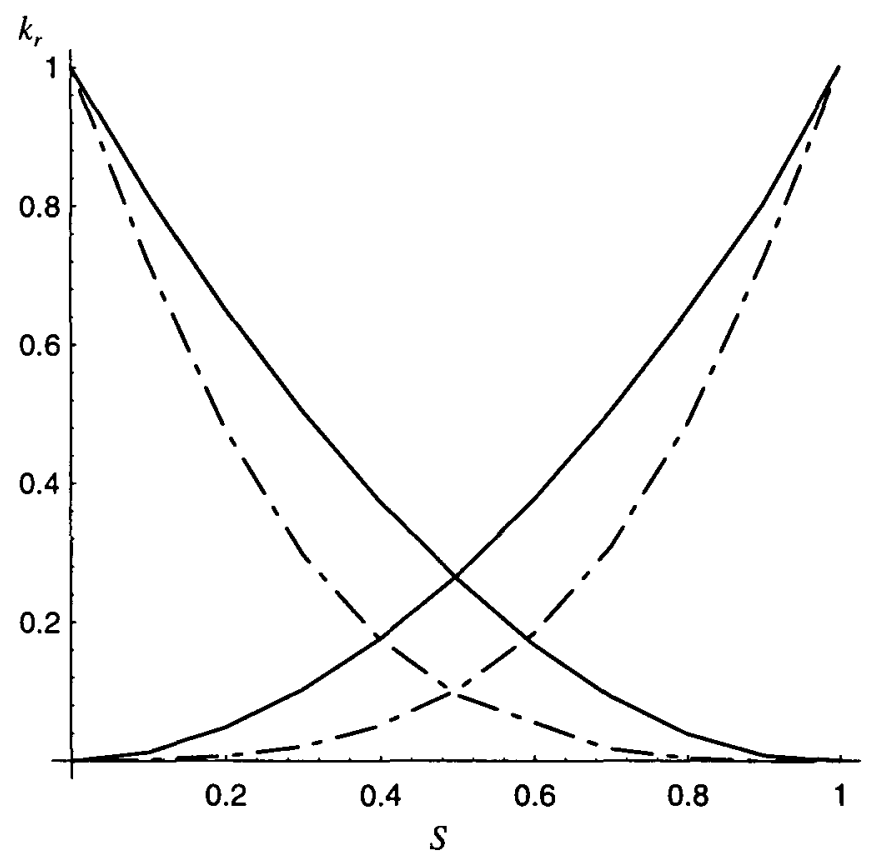

Figure 6. Relative permeability parallel to the bedding direction (solid lines) compared to perpendicular to the bedding (broken lines). Simulations used $\mathrm{fBm}$ with $H=0.5$ stretched by a factor of $2, L=17$ with averages over 1000 realisations.

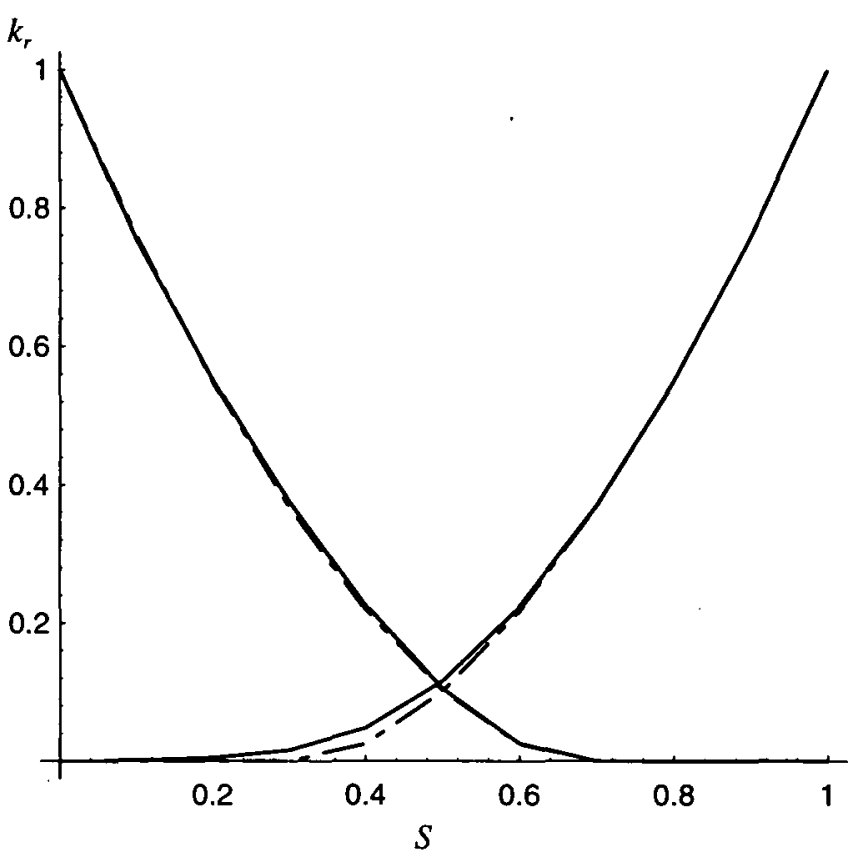

Figure 7. Invasion percolation compared with ordinary percolation on a network with uncorrelated properties. Little difference is observed. Difference is mainly for the invading phase at low saturations. Averages over 1000 realisations with $L=17$.

and perpendicular to the bedding in Berea sandstone cores. They observed that relative permeability at a given saturation is greater for flow parallel to the bedding than flow perpendicular to the bedding. This behaviour was reproduced using the invasion percolation model with correlated heterogeneity and anisotropy (Figure 6). For this example fractional Brownian motion with $H=0.5$ was stretched by a factor of 2 . Then simulations were performed both parallel and perpendicular to the bedding direction.

It is of interest to compare invasion percolation with ordinary percolation. There is little difference between the relative permeabilities calculated from invasion percolation and ordinary percolation on uncorrelated networks (Figure 7), although the initial breakthrough of the invading phase is very different. This observation was first noted by Sahimi [1993]. There is even less difference on correlated networks between the relative permeabilities (Figure 8). This observation suggests that subtle details of the network flow models may not be important for relative permeability calculations, although the details may be important for other applications. 


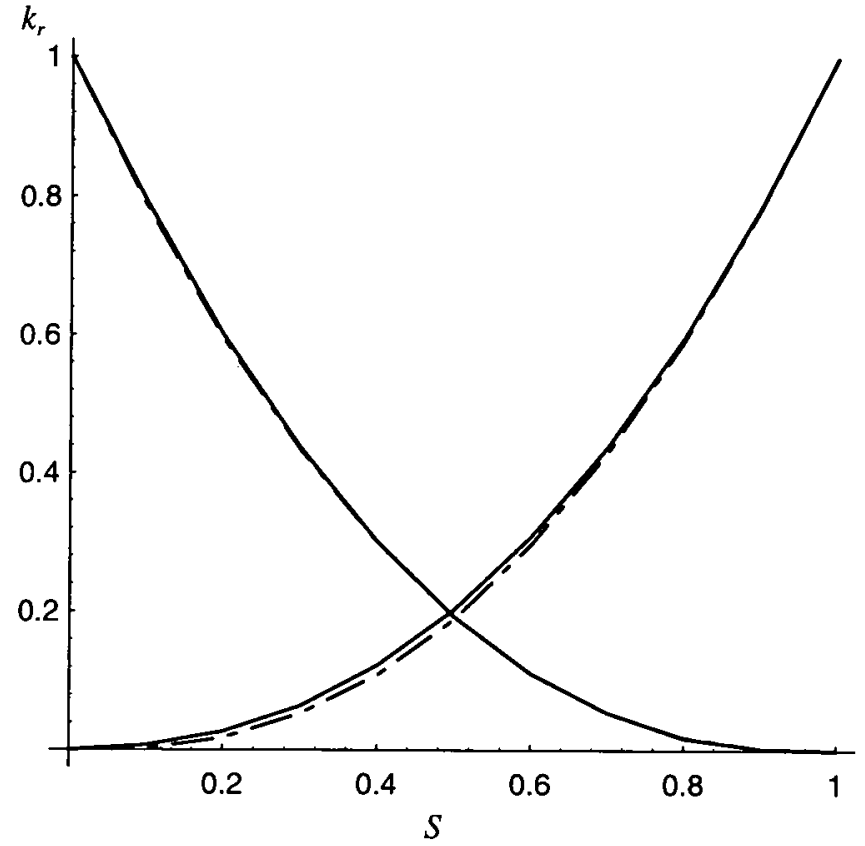

Figure 8. Invasion percolation compared with ordinary percolation on a network with properties derived from $\mathrm{fBm}$ with $H=0.25$. Even less difference is observed than for uncorrelated properties (Figure 5). Averages over 1000 realisations with $L=17$.

So far we have only considered average behaviour. The random processes used to create the network properties mean that each individual realisation is different to some degree. On an uncorrelated network, the variation between different realisations of relative permeability decreases with network size $L$ and individual realisations converge on the average. However, for fractional Brownian motion with $H=0.25$, the variability appears to remain constant in magnitude, independent of network size (Figure 9). This means that, if samples are taken from a porous rock with a variability described by for fractional Brownian motion, a fixed level of variability would be expected in the laboratory measurements. Alternatively, if the laboratory tests are selected to avoid variability, then a bias in the average estimate is possible. This concept is explored further in Figure 10.

For Figure 10, a single large simulation was performed on a $33 \times 33 \times 33$ network. Then four separate pieces were cut out of the network, rather like taking large samples from a reservoir. Relative permeabilities were then calculated for each of the pieces. Trapping was then turned on so that residual saturation could be investigated. Results are included

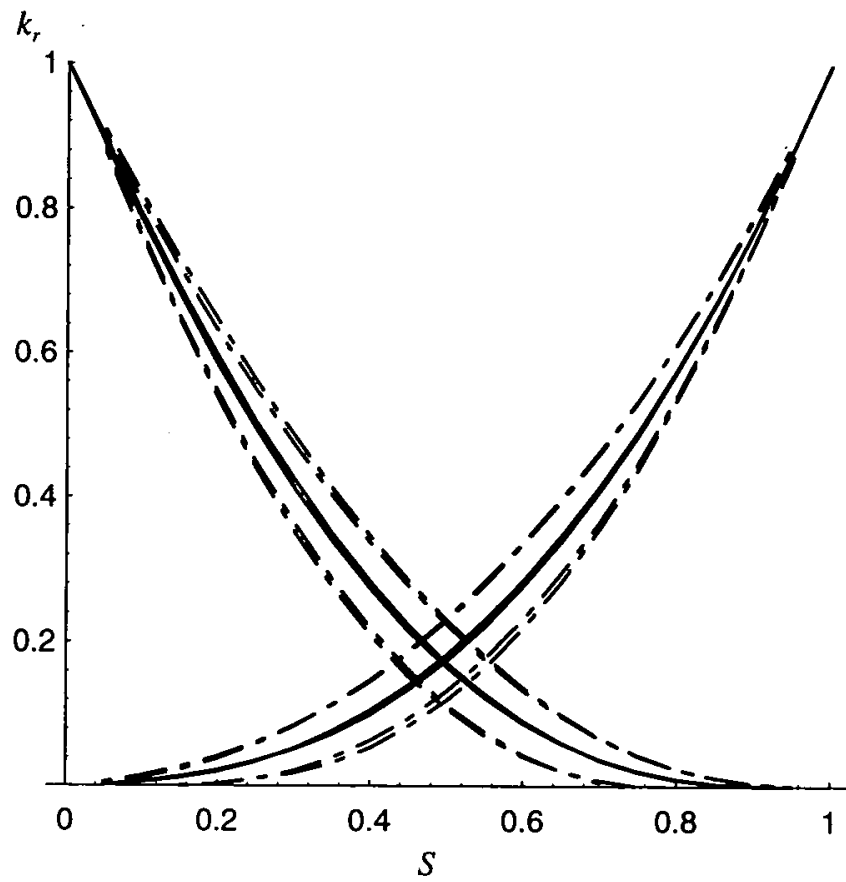

Figure 9. Effect of $L$ on relative permeability and variability in relative permeability. Solid lines are mean values, broken lines are \pm one standard deviation. Plot of $L=17$ is superimposed on $L=9$, showing little change with size $L$. Averages over 1000 realisations using $\mathrm{fBm}$ with $H=0.25$.

in Tables 5 for c correlated network and Table 6 for an uncorrelated network. The correlated network produced far more scatter, reproducing the variable behaviour of the values in Table 1.

Table 5. Residual saturation on a single $33 \times 33 \times 33 \mathrm{fBm}$ $H=0.25$ network, and values from simulations on four separate $16 \times 16 \times 16$ pieces of the same large network. The pieces have scattered values.

$\begin{array}{ll}\text { large network } & 0.35 \\ & \\ \text { piece 1 } & 0.24 \\ \text { piece 2 } & 0.38 \\ \text { piece 3 } & 0.32 \\ \text { piece 4 } & 0.35\end{array}$

We noted above that in our simple model, every pore is assigned identical volume, thus fraction of sites occupied in a percolation model becomes the fluid saturation. This 


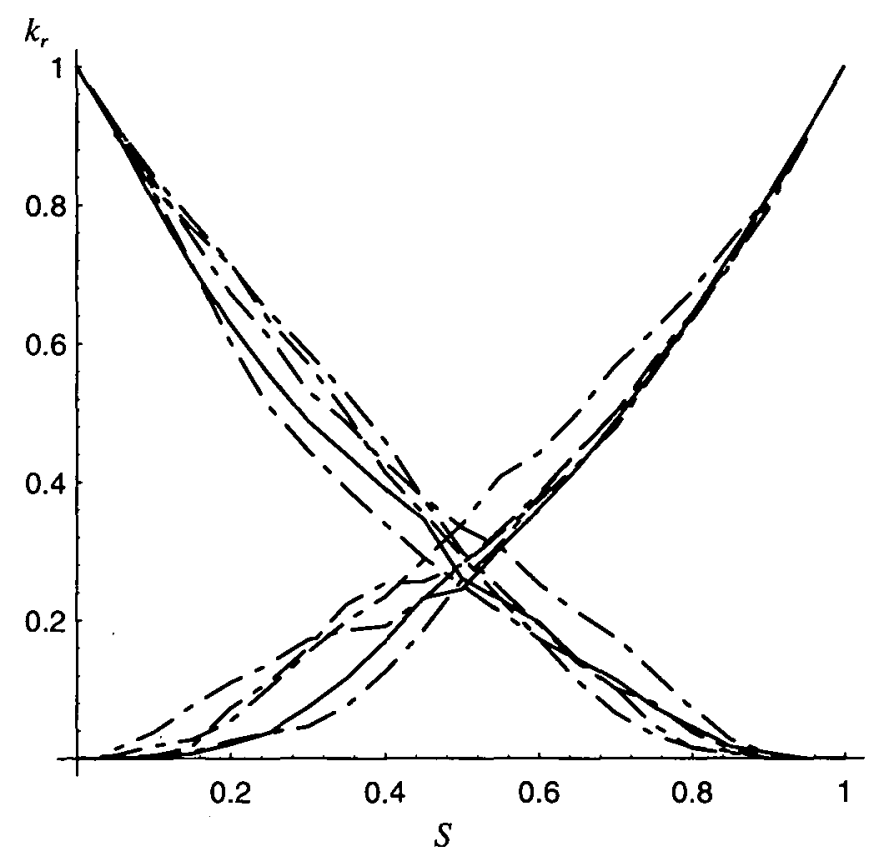

Figure 10. Effect of relative permeability on sample size and location. This corresponds to selecting "core" samples from a "reservoir." Solid lines are for a single large realisation with $L=33$. Broken lines are for 4 non-overlapping pieces using $L=16$ taken from the larger single realisation. Properties based on $\mathrm{fBm}$ with $H=0.25$ stretched by a factor of 4 .

Table 6. Residual saturation on a single $33 \times 33 \times 33$ uncorrelated network, and values from simulations on four separate $16 \times 16 \times 16$ pieces of the same large network. Scatter is much less than for the $33 \mathrm{fBm} H=0.25$ network (Table 5).

$\begin{array}{ll}\text { large network } & 0.34 \\ & \\ \text { piece 1 } & 0.34 \\ \text { piece 2 } & 0.35 \\ \text { piece 3 } & 0.35 \\ \text { piece 4 } & 0.36\end{array}$

assumption can be relaxed and the pores assigned different volumes. If the pore volumes are assigned values in the range $[0,1]$ matched to the throat radii, then an uncorrelated network gives the results in Figure 11. Similar movement of the relative permeability curves occurs when local conductivities are related to the throat sizes. These issues have been explored in detail by Jerauld and Salter [1990].

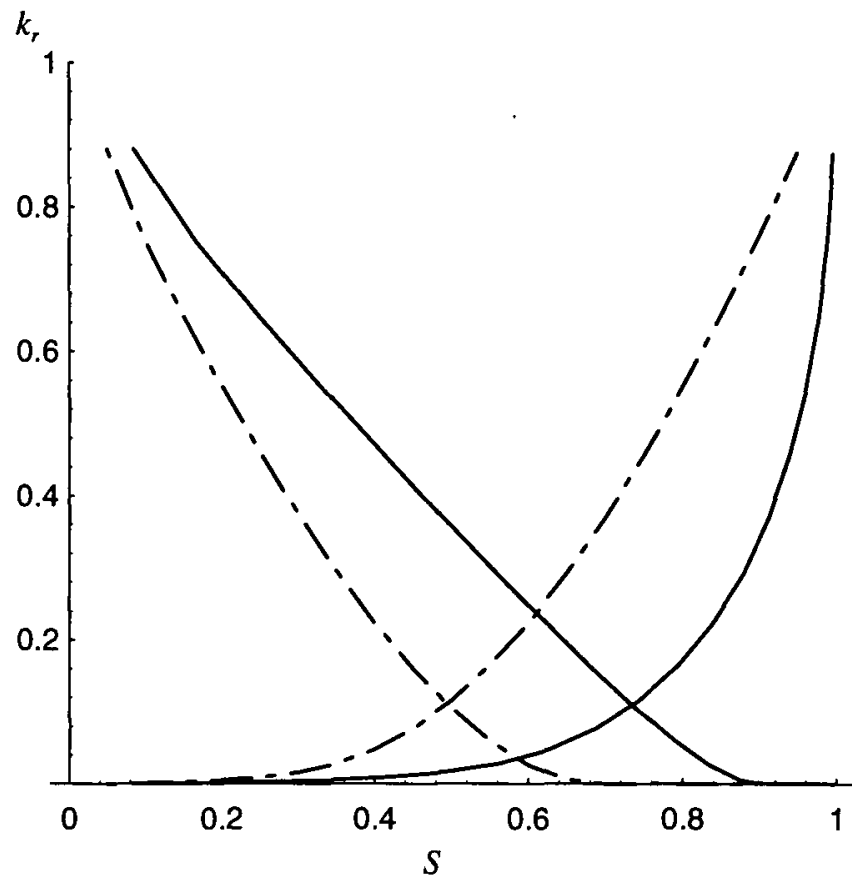

Figure 11. Effect of relationship between pore occupancy and saturation. In this example with uncorrelated properties, pores are assumed to have (a) volumes uniformly distributed between 0 and 1 (solid line) and (b) uniformly equal volumes (broken line).

\section{CONCLUSIONS}

We have investigated the consequences of introducing correlated heterogeneity into the invasion percolation model. The simple correlated network model has been able to reproduce the observation that relative permeability at a given saturation is greater for flow parallel to the bedding in comparison with flow perpendicular to the bedding. This model also appears to produce variability that is consistent over large changes in length scales.

Scope exists for simulations using more detailed pore network models. This includes both details in pore-scale physics and the description of the heterogeneity. For instance, scope exists for the simulation of cross-bedding, where the direction of anisotropy varies within the network. Two-component pore-throat models could also be implemented. 


\section{NOMENCLATURE}

$\begin{array}{ll}H & \text { Hurst parameter } \\ L & \text { network length, number of sites } \\ S & \text { invading phase saturation, fraction } \\ S_{b} & \text { invading phase saturation at breakthrough } \\ S_{r} & \text { defending phase residual saturation } \\ d & \text { scaling exponent } \\ k_{r} & \text { relative permeability, fraction } \\ \alpha & \text { Lévy index }\end{array}$

\section{REFERENCES}

Blunt, M., King, M.J. and Scher, H. (1992) Simulation and theory of two-phase flow in porous media. Phys. Rev. A 46, 7680-7699.

Blunt, M. and King, P. (1991) Relative permeabilities from two- and three-dimensional pore-scale network modelling. Transport in Porous Media, 6, 407-433.

Corey, A. T. and Rathjens, C. H. (1956) Effect of stratification on relative permeability. Trans. AIME, 207, 358-360.

Durlofsky, L.J. (1991) Numerical calculation of equivalent grid block permeability tensors for heterogeneous porous media. Water Resour. Res., 27, 699-708.

Heiba, A.A., Sahimi, M., Scriven, L.E. and Davis, H.T. (1992) Percolation theory of two-phase relative permeability. SPE Reservoir Engineering, 7, 123-132.

Hewett, T. (1986) Fractal distributions of reservoir heterogeneity and their influence on fluid transport, SPE 15836, presented at the SPE Annual Technical Conference \& Exhibition, New Orleans, 5-8 Oct.

Hirsch, L.M. and Thompson, A.H. (1995) Minimum saturations and buoyancy in secondary migration. AAPG Bulletin, 79, 696-710.

Honarpour, M.M., Cullick, A.S. Saad, N. and Humphreys, N.V. (1995) Effect of rock heterogeneity on relative permeability: Implications for scaleup. J. Petrol. Tech., 47, 980-986.

Hughes, B.D. (1996) Random Walks and Random Environments. Volume 2: Random Environments. Clarendon Press, Oxford.

Jerauld, G.R. and Salter, S.J. (1990) The effect of porestructure on hysteresis in relative permeability and capillary pressure: Pore-level modeling. Transport in Porous Media, 5, 103-151.

Johnson, E.F., Bossler, D.P. and Naumann, V.O. (1959) Calculations of relative permeability from displacement experiments. Trans. AIME, 216, 370-372.

King, P.R. (1989) The use of renormalisation for calculating effective permeability. Transport in Porous Media, 4, 37-58.
Larson, R.G., Davis, H.T. and Scriven, L.E. (1981) Percolation theory of two-phase flow in porous media. Chem. Eng. Sci., 36, 57-73.

Larson, R.G., Scriven, L.E. and Davis, H.T. (1977) Percolation theory of residual phases in porous media. Nature, 268, 409-413.

Meakin, P. (1987) Diffusion-limited aggregation on multifractal lattices: A model for fluid-fluid displacements in porous media. Phys. Rev. A., 36, 2833-2837.

Painter, S. and Paterson, L. (1994) Fractional Lévy motion as a model for spatial variability in sedimentary rock. Geophys. Res. Lett., 21, 2857-2860.

Painter, S. (1995) Random fractal models of heterogeneity: The Lévy-stable approach. Math. Geology, 27, 813-830.

Painter, S., Paterson, L. and Boult, P. (1995) Improved technique for stochastic interploation of reservoir properties, SPE 30599, presented at the SPE Annual Technical Conference \& Exhibition, Dallas, 22-25 Oct.

Painter, S. (1996a) Evidence for non-Gaussian scaling behavior in heterogeneous sedimentary formations. Water Resour. Res., 32, 1183-1195.

Painter, S. (1996b) Stochastic interpolation of aquifer properties using fractional Lévy motion. Water Resour. Res., 32, 1323-1332.

Painter, S. (1996c) New algorithm for conditional simulation of Lévy fractal fields, submitted.

Pathak, P., Davis, H.T. and Scriven, L.E. (1982) Dependence of residual nonwetting liquid on pore topology, SPE 11016, presented at the SPE Annual Technical Conference \& Exhibition, New Orleans, 26-29 Sept.

Pereira, G.G., Pinczewski, W.V., Chan, D.Y.C., Paterson, L. and Oren, P.E. (1996) Pore-scale network model for drainage-dominated three-phase flow in porous media. Transport in Porous Media, in press.

Pickup, G.E. and Sorbie, K.S. (1994) Development and application of a new two-phase scaleup method based on tensor permeabilities, SPE 28586, presented at the SPE Annual Technical Conference \& Exhibition, New Orleans, 25-28 Sept.

Sahimi, M. (1993) Flow phenomena in rocks: From continuum models to fractals, percolation, cellular automata, and simulated annealing. Rev. Modern Physics, 65, 1393-1534.

Saupe, D. (1988) Algorithms for random fractals. In: The Science of Fractal Images, H.-O. Peitgen \& D. Saupe (Eds.) Springer-Verlag, New York Ch. 2.

Wilkinson, D. and Willemsen, J.F. (1983) A new form of percolation theory. J. Phys. A: Math. Gen. 16, 3365 3376. 
314 